\title{
Neue regionale Entwicklungskonzepte für periphere ländliche Räume
}

Das Beispiel der schottischen Highlands \& Islands ${ }^{1}$

\section{New regional development concepts for rural peripheries}

The case of the Scottish Highlands \& Islands

\begin{abstract}
Kurzfassung
Die schottischen Highlands and Islands gelten seit langem als einer der klassischen Peripherräume Europas. Vor diesem Problemhintergrund vermittelt der Beitrag zunächst einen kurzen Überblick über die historische Entwicklung der Highlands and Islands, wobei der Schwerpunkt auf den Prozess der „Peripherisierung“ der Region und die Entwicklung und Ausgestaltung regionalpolitischer Fördermaßnahmen während der letzten 40 Jahre gelegt wird. Ein weiterer Fokus liegt auf der gegenwärtigen Umgestaltung von Konzepten, Programmen und Instrumenten der Regionalentwicklung, in deren Zentrum die skizzierte Diskussion um Konzepte eines integrated rural development (integrierte ländliche Entwicklung) steht.
\end{abstract}

\begin{abstract}
The Scottish Highlands and Islands have been classified as one of the classic rural peripheries within Europe for a long time. Against this background the paper summarizes the historical development and the process of marginalisation the Highlands and Islands have experienced, in particular describing the regional policy measures applied over the last 40 years. Further focus lies on the current discussion of so called integrated rural development concepts that form the core of a more general reform of concepts, programmes and instruments in Scottish regional policy.
\end{abstract}

\section{Die schottischen Highlands and Islands - eine klassische Peripherie Europas im Umbruch}

Die Bezeichnung Highlands and Islands, häufig auf den Begriff der Highlands verkürzt (im Deutschen entsprechend Schottisches Hochland), ist nicht eindeutig definiert. Häufig wird darunter der geomorphologische Großraum des festländischen Berglandes nördlich der mittelschottischen Senke zwischen Glasgow und Edinburgh verstanden. Gleichzeitig findet der Begriff für die Gebiete Verwendung, die 1886 als sog. crofting counties definiert wurden. Ferner wird mit dem Begriff das Gebiet der 1975 geschaffenen administrativen Einheit des Highland Council bezeichnet, das neben den festländischen Teilen Nordschottlands auch die Insel Skye umfasst. Als Träger der Regionalentwicklung, insbesondere der regionalen Wirtschaftsförderung, wurde 1965 das Highlands and Islands Development Board (HIDB) gegründet, dessen Arbeitsgebiet neben den Highlands auch die Inselgruppen der Shetlands, Orkneys und Western Isles umfasste. Heute ist die Nachfolgeorganisation Highlands and Islands Enterprise (HIE) Träger der Regionalentwicklung in diesem Raum. Dieser soll hier als Highlands and Islands bezeichnet werden. 
Die Highlands and Islands sind in ihrer Gesamtheit als ein Gebirgsraum zu charakterisieren. Ein weiteres Charakteristikum ist ihre extreme periphere verkehrsräumliche Lage, sowohl innerhalb Schottlands, Großbritanniens als auch Europas. Insbesondere die Inselgruppen sind durchweg sieben bis zehn Autostunden von der nächsten Großstadt entfernt, wobei das Straßennetz vor allem an der Westküste und auf den Inseln nach wie vor vielfach durch einspurige Regionalstraßen geprägt wird. Viele Inseln sind zudem nur mittels eingeschränkter Fährverbindungen mit dem Festland bzw. benachbarten Inseln verbunden. Entsprechend kommt dem Luftverkehr wachsende Bedeutung für die Sicherstellung der Mobilität zu.

Eine Besonderheit des Schottischen Hochlandes ist ferner die relativ hohe Anzahl aktiver Sprecher der Regionalsprache Gaelic; jüngste Erhebungen gehen dabei von ca. 80000 Menschen aus (entsprechend gut $18 \%$ der Bevölkerung). Sie konzentrieren sich auf den Nordwesten der Hochlandgebiete, vornehmlich auf die Insel Skye sowie auf die Western Isles.

Neben dem skandinavischen Norden zählen die Highlands and Islands zu den am dünnsten besiedelten Regionen Europas. Mit einer Gesamtbevölkerung von 430181 Einwohnern (2001) beläuft sich die durchschnittliche Bevölkerungsdichte auf $10,7 \mathrm{E} / \mathrm{km}^{2}$. An der Nordwestküste sowie auf einigen Inseln liegt der Wert noch deutlich darunter (Skye and Lochalsh: $4,4 \mathrm{E} / \mathrm{km}^{2}$ ). Die Bevölkerungszahl entspricht $8,4 \%$ der Gesamtbevölkerung Schottlands bei einem Flächenanteil von $51,8 \%$ der Landesfläche.

Die geringe Bevölkerungdichte hängt eng zusammen mit dem langen Prozess der systematischen Peripherisierung des Hochlandes und den daraus resultierenden sozialen und ökonomischen Problemen. Frühe Wurzeln dieser Entwicklung sind bereits in dem mittelalterlichen Prozess der schottischen Staatsbildung zu finden, in dessen Verlauf die Highlands and Islands sukzessive durch den neuen Zentralstaat unterworfen wurden; bis Ende des 15. Jahrhunderts hatten dabei alle Hochlandgebiete, zuletzt das Lordship of the Isles (1493), ihre vormalige Autonomie verloren. Nach Hunter (2000, S. 144 ff.) hatte der Prozess der territorialen Integration de facto den Charakter einer Kolonisierung, die insbesondere in der zunehmenden Missachtung, schließlich auch Unterdrückung der gälischen Sprache und Kultur durch die herrschende Elite in Edinburgh Ausdruck fand. Noch weiter reichende Konsequenzen ergaben sich aus der staatlichen Vereinigung von Schottland und England im Jahre 1707. Als Folge der von mehreren erfolglosen Aufständen im Lande (zuletzt 1746) begleiteten Vereinigung wurde das bis dahin charakteristische clan-
System, territorial organisierte Sippenverbände bzw. Stämme, im Hochland verboten und der traditionelle Gemeinbesitz der clans zunächst englischer Verwaltung unterstellt, 1780 jedoch an die clan-chiefs zurückgegeben. Anders als zuvor wurden diese nun jedoch nicht mehr als Führer ihrer clans sondern als Feudalherren mit allen grundherrlichen Rechten nach englischem Vorbild eingesetzt. Das Bestreben der Grundherren nach einer wirtschaftlich profitablen Entwicklung ihrer Landgüter führte zur großflächigen Umstellung der Agrarstruktur vom Ackerbau auf die extensive Schafbeweidung. Da der Einrichtung großer Schaffarmen die überkommene Landwirtschaft im Wege stand, wurden umfangreiche Programme zur Umsiedlung der kleinbäuerlichen Bevölkerung aus den Hochlandgebieten an die Küste entwickelt, wo diesen kleine Parzellen Land, sog. crofts, zur Bewirtschaftung zugewiesen wurden. Beginnend um 1810* wurden die als Highland Clearances bekannt gewordenen Umsiedlungsaktionen der Bauern, häufig zwangsweise und unter Einsatz militärischer Gewalt, bis in die 1870er Jahre in allen Teilen der Highlands and Islands durchgeführt. Als Folge der starken Übervölkerung der Küstensäume sowie der geringen wirtschaftlichen Entwicklungsmöglichkeiten ihrer crofts war die kleinbäuerliche Bevölkerung lange Zeit Not und Elend ausgesetzt. Mehrere gravierende Hungersnöte unterstreichen die Dramatik der Entwicklung. Große Teile der Bevölkerung waren somit zur Abwanderung in die schottischen und englischen Industriereviere, in beträchtlichem Umfang auch nach Übersee (USA, Kanada usw.) gezwungen. Erst 1886 wurde eine gesetzliche Grundlage geschaffen, die die Rechte der crofter und Grundherren gegeneinander abgrenzte und die existierenden kleinbäuerlichen Siedlungen in ihrem Bestand absicherte. Gleichwohl waren die crofter zur Entwicklung zahlreicher zusätzlicher Erwerbsmöglichkeiten gezwungen, um ihre Existenz zu sichern (Fischfang, Textilherstellung, Seetangverarbeitung usw.) (vgl. Heineberg 1997, S. 83 ff.).

Infolge der langjährigen Umsiedlung, Ab- und Auswanderung erlitten die inneren Hochlandgebiete z.T. extreme Bevölkerungsverluste, während die Zielgebiete der Wanderung, z.B. die Western Isles und andere Inseln, deutliche Zunahmen erfuhren. Die heutige Situation ist jedoch nur noch zum Teil durch die historischen Wanderungen zu erklären, sie ist vor allem Spiegel der jüngeren wirtschaftlichen Entwicklung in den Highlands and Islands und von regionalpolitischen Maßnahmen zur Förderung der Hochlandgebiete, die nicht ohne Auswirkungen auf die Bevölkerungsentwicklung und -verteilung blieben. 
Bis in die 1960er und 1970er Jahre war die Situation in großen Teilen der Highlands and Islands, insbesondere an der Nordwestküste, auf Skye und den Western Isles, durch eine breite wirtschaftliche Stagnation, fehlende Beschäftigungsmöglichkeiten, niedrige Löhne und relativ hohe Arbeitslosenquoten sowie einen allgemeinen Verfall der privaten und öffentlichen Infrastruktur (Verkehr, Bildung, Kultur usw.) bestimmt. Mit der daraus resultierenden Abwanderung vor allem junger Menschen waren zudem eine Überalterung vieler Ortschaften sowie ein Verlust des Gemeinschaftsgefühls und der Bereitschaft zum aktiven Handeln verbunden, was einer sozialen Isolierung dieser Gebiete gleichkam.

Um einer weiteren Entvölkerung der Highlands and Islands entgegenzuwirken und gezielte Maßnahmen zu einer wirtschaftlichen und sozialen Reaktivierung der Region einzuleiten, wurde 1965 das HIDB installiert. Dies kann als der Beginn einer gezielten Regionalförderung für die Problemgebiete des schottischen Nordens angesehen werden, wenngleich schon Ende der 1940er Jahre erstmals ein Fördergebiet im Hochland ausgewiesen worden war. Die Einrichtung des $H I D B$ stellt insofern eine Zäsur dar, als damit auf der Grundlage eines eigenen Gesetzes eine zentrale Behörde geschaffen wurde, der weitreichende Kompetenzen und eigene finanzielle Mittel für die regionale Wirtschaftsförderung übertragen wurden. Vorrangiges Ziel der Behörde sollte dabei sein, die ökonomische Entwicklung der Hochlandgebiete systematisch auf eine breitere Basis $\mathrm{zu}$ stellen. Hierunter wurde zunächst primär die Ansiedlung von großen Industrien und die Schaffung industrienaher Infrastruktur verstanden, die der Bevölkerung außerhalb der Kleinpächter-Landwirtschaft neue Einkommens- und Existenzmöglichkeiten eröffnen sollten. Zudem wurde der Gründung des $H I D B$ vielfach auch eine hohe symbolische Bedeutung beigemessen: als erster Versuch einer „Wiedergutmachung“ für die jahrhundertelange Ausplünderung der Highlands and Islands durch den schottischen bzw. den britischen Staat (vgl. Hunter 2000, S. 355 ff.; Turnock 1974, S. 39 ff.).

Unzweifelhaft stellte der Beginn einer systematischen Wirtschaftsförderung in den 1960er Jahren einen Wendepunkt für die Entwicklung der Highlands and Islands dar. Bis auf wenige Ausnahmen (Wasserkraftwerke, Aluminiumschmelze) war das Schottische Hochland bis dahin weitgehend unindustrialisiert. Erst als Folge des Baus eines Atomkraftwerkes in Caithness rückte die Region zunehmend in den Blickpunkt von Gewerbe und Industrie. Um Fort William sowie vor allem an der Ostküste, um Inverness und den Moray Firth, begannen sich Ansätze einer Industriekonzent- ration zu entwickeln. Das übrige Hochland blieb von dieser Entwicklung jedoch ausgeschlossen. Eine breitere Entwicklung entfaltete im Vergleich dazu die Förderung traditioneller ländlicher Gewerbezweige, die an verschiedenen Orten zum Ausgangspunkt einer ökonomischen Reaktivierung wurden. Hierzu zählen insbesondere das Textilgewerbe (Strickerei- und Webereiprodukte, z. B. „Harris Tweed“) sowie die Fischereiwirtschaft (Fischfang, Fischzucht und Fischverarbeitung), ferner die Whiskyherstellung, die eine nachhaltige (Wieder)Belebung erfuhren (vgl. Wehling 1987, S. 19 ff.; Turnock 1974, S. 18 ff.).

Maßgeblich für die jüngere wirtschaftliche Entwicklung war jedoch der Aufschwung zwei weiterer - neuer - Wirtschaftszweige während der 1970er und 1980er Jahre: die Erdöl- und -gasförderung sowie der Tourismus. Erstere ist heute für die Highlands and Islands von zentraler ökonomischer Bedeutung. Seit gegen Ende der 1960er Jahre an der englischen und vor allem schottischen Nordseeküste ergiebige Offshore-Erdölund Erdgasvorkommen entdeckt wurden, hat sich dieser Wirtschaftszweig bis zur Mitte der 1980er Jahre geradezu explosionsartig entwickelt. Hiervon profitierte nicht nur die Öl- und Gasindustrie, sondern es kam auch zu vielfältigen Auswirkungen auf andere Wirtschaftszweige (Plattformwerften, Ausrüstungs- und Versorgungsunternehmen, Tanklager, Hafenwirtschaft usw.). Beträchtlich waren ebenso die Effekte auf den regionalen Arbeitsmarkt, wobei die größten Anteile an Beschäftigten auf den Nordosten Schottlands entfallen; in den anderen Teilen der Highlands and Islands fallen diese deutlich geringer aus (vgl. Heineberg 1997, S. $238 \mathrm{ff}$.).

$\mathrm{Zu}$ den dynamischen Wachstumssektoren zählt schließlich auch der Tourismus. Seit Anfang der 1980er Jahre hat dieser einen kontinuierlichen Aufschwung genommen, der sich nicht zuletzt auch der systematischen Förderung durch den $H I D B$ verdankt. Landschaftliche Attraktionen, insbesondere das Bergland und die Küste, historische Denkmäler (Schlösser, Burgen) sowie soziokulturelle Besonderheiten (Highland Games, Whisky) gelten als Hauptanziehungspunkte des nationalen wie internationalen Schottlandtourismus. Während sich die Entwicklung des Tourismus zunächst auf Gebiete des festländischen Hochlandes konzentrierte, sprang diese in der Folge auch auf einzelne relativ festlandsnahe Inseln (z.B. Arran, Mull, Skye, Shetland Isles) über. Wesentlich langsamer kann dagegen die äußerste Peripherie der Inselgebiete (Western Isles) in die touristische Entwicklung integriert werden (vgl. Wehling 2000).

Ausrichtung und Ergebnisse der skizzierten Förderpolitik wurden frühzeitig zum Gegenstand einer kon- 
troversen Diskussion, die mit sehr unterschiedlichen Bewertungen zur Rolle des HIDB einhergingen. Während die vorrangig auf die Anforderungen der Wirtschaft ausgerichteten Fördermaßnahmen des $H I D B$ einerseits als Ansatz zur ökonomischen Reaktivierung eines besonders benachteiligten Peripherraumes Anerkennung erfuhren, wurden diese andererseits als angesichts der komplexen Problemlagen des schottischen Hochlandes als unzureichend kritisiert (vgl. Shucksmith 1998, S. 5). Tatsächlich hat die Tätigkeit des $H I D B$ nicht verhindern können, dass die Entwicklung in den Highlands and Islands teilräumlich sehr unterschiedlich verlief. Insbesondere die ländlichen Gebiete des Nordwestens und viele Inseln wurden in diesem Zusammenhang als "Verlierer" dieser Politik gesehen, während umgekehrt die Erfolge der Wirtschaftsförderung weitgehend auf Standorte an der Nordostküste, schwerpunktmäßig den Großraum Inverness, beschränkt blieben. Dies spiegelt sich auch im Muster der Bevölkerungsentwicklung wider. Während diese allgemein seit Mitte der 1960er Jahre erstmals wieder eine Zunahme erfahren hat, zeigt die Entwicklung in den unterschiedlichen Teilgebieten des Hochlandes jedoch ein differenzierteres Bild. Die größten Zuwächse verzeichneten danach die Gebiete an der Nordostküste, deutlich niedriger waren dagegen die Zuwächse im Westen. Auf dem Lande verlief die Entwicklung generell zugunsten der größeren Hauptorte, während viele kleine, vor allem entlegene Siedlungen Bevölkerung verloren. Dies trifft insbesondere auf die Western Isles zu, die noch bis heute anhaltende Bevölkerungsverluste $\mathrm{zu}$ verzeichnen haben (vgl. Turnock 1974, S. 29 ff; Hunter 2000, S. 365 ff.).

Ein fundamentaler Wandel hinsichtlich der Rolle des $H I D B$ vollzog sich im Verlauf der 1980er Jahre. Den Hintergrund dieser Entwicklung bildeten die grundsätzlichen Vorbehalte der konservativen britischen Regierung Thatcher (später Major) gegenüber jeglichen Formen der interventionistischen Politik. Diese wurden schon bald auch gegenüber dem $H I D B$ geltend gemacht (vgl. Hunter 2000, S. 370/371). Ausdruck dieser Entwicklung ist eine „Korrektur" der HIDB-Politik, als deren vorrangiges Ziel nunmehr die Aktivierung privaten unternehmerischen Kapitals definiert wurde (vgl. Shucksmith 1998, S. 5). Diese Hinwendung zu einer dezidiert marktorientierten Regionalpolitik stellte jedoch nur den letzten Schritt vor der Auflösung der Behörde und deren völliger Neuorganisation im Rahmen von $H I E$ dar, die im Jahr 1991 vollzogen wurde (vgl. Fairly/Lloyd 1998, S. 203 ff.). Seither ist HIE Träger der schottischen Regionalpolitik in den Highlands and Islands. Es handelt sich dabei um eine sog. Quango, eine quasi-autonomous non-governmental organization, die funktional zwischen der Zentral- regierung und den lokalen Verwaltungsebenen angesiedelt ist. Entsprechende Organisationsstrukturen wurden inzwischen für zahlreiche vormals staatliche Aufgabenbereiche in Großbritannien geschaffen. Der Begriff wird dabei zunehmend diffus verwendet und subsumiert ",anything and everything that occupies the terrain between the public and private sectors and thereby includes a wide range of bodies that have widely different powers, responsibilities and relations with central government" (Greenwood u. a. 2002, S. 152).

\section{Integrierte ländliche Entwicklung - Neues Leitbild der schottischen Regionalpolitik}

Für die gegenwärtige Diskussion um die Ausgestaltung der schottischen Regionalpolitik, insbesondere der Förderpolitik für die Highlands and Islands, kann in mehrfacher Hinsicht von einer Phase der Neuausrichtung gesprochen werden. Vielfach ist sogar von „Experimenten " die Rede, die einerseits als Ausdruck der Innovationskraft, andererseits der Unübersichtlichkeit des gegenwärtigen Status der regionalpolitischen Debatte interpretiert werden können.

Im Hinblick auf die zukünftige programmatische Ausrichtung der Förderpolitik für die Highlands and Islands ist dabei ein Baustein von besonderem Interesse: die Diskussion um Konzepte, Strategien und Instrumente einer integrierten ländlichen Entwicklung. Diese bleibt nicht unbeeinflusst vom fortschreitenden Prozess der formalen Erneuerung verschiedener regional wirksamer Politikbereiche, insbesondere auch der regionalen Wirtschaftsförderung, wie sie bereits zu Anfang der 1990er Jahre in Schottland eingeleitet wurde.

Eine weitere Akzentuierung hat diese schließlich durch den Prozess der devolution, der Dezentralisierung Großbritanniens mit dem Ziel des Aufbaus einer föderativen Staatsstruktur, erfahren. Von besonderer Bedeutung ist hierbei das schottische Referendum von 1997, in dessen Folge Schottland seit 1999 (Konstituierung des schottischen Parlamentes) mit einem deutlich höheren $\mathrm{Ma} ß$ an Selbstverwaltung ausgestattet worden ist. Somit eröffnen sich weitere politische Gestaltungsräume einer „eigenständigen Schottlandpolitik", die es bisher in dieser Form nicht gegeben hat.

Das Leitbild einer integrierten ländlichen Entwicklung steht im engen Zusammenhang mit wachsenden Zweifeln an der Wirksamkeit der Regionalpolitik und ihrer Instrumente, wie sie bereits seit Ende der 1970er Jahre auf nationaler wie europäischer Ebene diskutiert wurden. Im Vordergrund stand dabei die Ergänzung des etablierten Förderinstrumentariums durch eine Reihe neuartiger Programme, die auf eine gezielte und bes- 
sere Förderung (peripherer) ländlicher Räume zielen sollten. Neben anderen Begriffen spielte dabei auch der der integrierten ländlichen Entwicklung eine zentrale Rolle. Wenngleich dieser keineswegs eindeutig definiert und nach wie vor Gegenstand der regionalpolitischen wie regionalwissenschaftlichen Diskussion ist, lassen sich einige Elemente benennen, die für die konzeptionelle Grundlegung und praktische Ausgestaltung entsprechender Handlungsansätze von zentraler Bedeutung sind.

In Anlehnung an Shortall/Shucksmith (1998), Shucksmith (1998) und Marsden / Bristow (2000) werden unter integrated rural development Konzepte zur Förderung insbesondere peripherer ländlicher Räume verstanden, die

- auf die Entwicklung sektor- bzw. ressortübergreifender Handlungsansätze ausgelegt sind,

- mit einer Verlagerung von vormals zentral angelegten politischen Entscheidungsstrukturen auf die regionale und lokale Ebene verbunden sind,
- eine gezielte und durchgängige Partizipation der betroffenen Bevölkerung „vor Ort“ vorsehen,

- auf den konsequenten Aufbau und die Nutzung von Netzwerken privater, öffentlicher und ehrenamtlicher Akteure bauen und

- mit einer Implementierung geeigneter Formen der regionalen Aktivierung, Regionalberatung und des Regionalmanagements verbunden sind.

Wichtige Impulse für die Verankerung dieser Vorstellungen gingen von der europäischen Ebene aus. $\mathrm{Zu}$ nennen ist hier u.a. die sog. „Erklärung von Cork" aus dem Jahre 1996 zur zukünftigen Ausrichtung der Politik für die ländlichen Räume, die sich ausdrücklich zu einer integrierten ländlichen Entwicklungspolitik bekennt. In Schottland waren es vor allem mehrere sog. Rural White Papers des Scottish Office in den 1990er Jahren, in denen die Forderung nach einer veränderten ländlichen Entwicklungspolitik im Sinne integrierter Handlungsansätze verstärkt ihren Ausdruck fand. Besonderes Gewicht wird in diesen Dokumenten auf

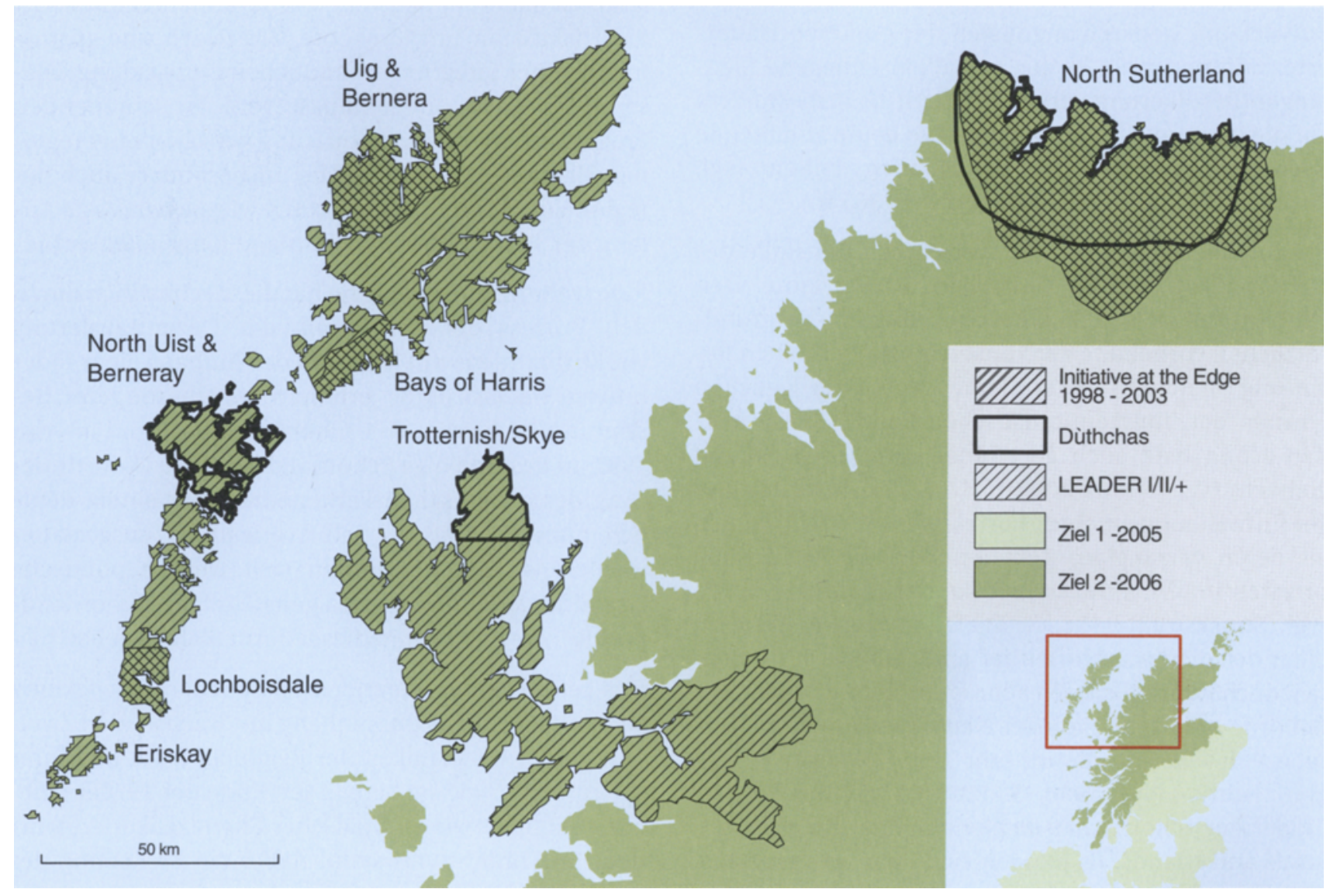

Quelle: eigene Bearbeitung 
die Forderung nach einem partizipativen, netzwerkorientierten lokalen Ansatz ländlicher Entwicklung gelegt, dessen Ziel es sein soll, „for individuals and groups to take more responsibility for their own development, overcoming the corrosive psychological effect of decades of lack of control and the promotion of negative self-images. A grass-roots, bottom-up approach to sustainable rural development is likely to be a relatively slow process - there are no instant solutions - but it is a more durable approach because it has its roots firmly planted in rural communities themselves" (Scottish Officel997 in Shucksmith 1998, S. 2).

Zeitgleich wurden erstmals auch entsprechende Schritte zur praktischen Implementierung eines solchen "neuen“ lokalen Entwicklungsansatzes unternommen. Eine gewisse Initialwirkung hatte diesbezüglich der 1996 eingerichtete Scottish Rural Partnership Fund mit mehreren spezifischen Fördertöpfen (2001 bestätigt und erweitert). Danach sollen Behörden, Verbände, Wirtschaft und Bürger in Local Rural Partnerships zusammengeführt werden mit dem Ziel, die lokale bzw. regionale Beteiligung bei Planungen zu gewährleisten („gain community-level representation“) und darüber hinaus lokale Akteure $\mathrm{zu}$ animieren, eigene Projekte und Initiativen ins Leben zu rufen (,facilitate community capacity building") (Brown 2000, S. 1).

Zentraler Einfluss auf die praktische Implementierung und Ausgestaltung einer solchen integrierten ländlichen Entwicklung wird insbesondere auch der Wirkung der europäischen Strukturfonds zugeschrieben. Mit diesen ist die Finanzierung vieler Projekte überhaupt erst möglich geworden, auch von solchen, die sonst keine nationale Förderpriorität genossen hätten. Spezielle Bedeutung erlangte die EU-Gemeinschaftsinitiative LEADER für Schottland (seit 1991) (vgl. Black/Conway 1996). Bekanntermaßen gilt das LEADER-Programm als „Vorbild“ für einen integrierten Ansatz ländlicher Entwicklung. Wenn die Praxis auch eine Reihe von Defiziten erkennen lassen hat, so kann gerade in Schottland der Wert von LEADER als Katalysator für eine zunehmend breitere Diskussion um entsprechende Handlungsansätze und deren praktische Erprobung nicht hoch genug angesiedelt werden. Dies gilt primär für den damit verbundenen programmatischen Input. Zu nennen ist hier vor allem die Vorbildwirkung der sog. Local Action Groups als Träger von LEADER, deren Konstruktion auf verschiedene andere Programme übertragen wurde. Weiterhin lässt sich mit der Gründung der Community Economic Development Partnerships eine unmittelbare Einflussnahme von LEADER auf die programmatische Ausrich- tung einer Schlüsselinstitution (Highland and Islands Enterprise) nachweisen.

Ebenso korrespondiert die Hinwendung zu Ansätzen einer integrierten ländlichen Entwicklung mit dem oben skizzierten Prozess der formalen Erneuerung und Umgestaltung im Bereich verschiedener regional ausgerichteter Politikbereiche, der sich seit Beginn der 1990er Jahre kontinuierlich weiter fortgesetzt hat. In Schottland betrifft dies insbesondere die organisatiorische Verankerung und programmatische Ausrichtung von $H I E$ als Nachfolgeorganisation des $H I D B$ (vgl. Kap. 3.1). Weiterhin ist die jüngere Neustrukturierung der ministeriellen Zuständigkeiten im Umfeld der Regionalentwicklung von Bedeutung. So kam es nach der Einrichtung des Scottish Executive als dezentraler schottischer Regierung im Jahre 1999 zu einer Reihe von veränderten Ressortzuschnitten. Hierzu gehört insbesondere das neu geschaffene Scottish Executive Environment and Rural Affairs Department (SEERAD), das zumindest im Titel den ablaufenden Paradigmenwechsel deutlich widerspiegelt. Nach wie vor wird die praktische Arbeit des Ministeriums allerdings durch eine überwiegend sektoral orientierte Agrarpolitik dominiert, für die es in Schottland eine sehr starke Lobby gibt. Weitere ministerielle Umstrukturierungen betreffen die Zuständigkeit für die Umsetzung der europäischen Förderprogramme, die unter den Verantwortungsbereich des Scottish Executive Development Department (SEDD) fallen, sowie für ausgewählte regionalpolitische "Experimente“, u.a. des Scottish Executive Life Long Learning Department (SELLD), das de facto Aufgaben eines Wirtschaftsministeriums erfüllt. Auch hierin drückt sich eine zunehmende Hinwendung zur Vorstellung von querschnittsorientierten, sektorübergreifenden Politikansätzen aus, auch wenn diese in der Praxis bislang erst bedingt wirksam werden.

Die neuen Ansätze kommen schließlich auch zunehmend in zahlreichen räumlich begrenzten, vor allem an der Peripherie der Highlands and Islands, den "most fragile areas", erprobten Modellvorhaben einer integrierten ländlichen Entwicklung zum Tragen. Diese neuartigen Programme unterscheiden sich von früheren Förderansätzen nicht nur durch diesen dezidiert territorialen Ansatz, der auf der Festlegung ausgewählter Fördergebiete beruht, sondern sind dabei ausdrücklich auch auf eine kleinräumige, lokale Maßstabsebene zugeschnitten, die mehr und mehr als die adäquate Handlungsebene regionalpolitischer Fördermaßnahmen erachtet wird (vgl. Kap. 3.2). 


\section{Regionalentwicklung als „Experiment“?}

Seit mindestens vier Jahrzehnten stehen die schottischen Highlands and Islands im Fokus regionalpolitischer wie regionalwissenschaftlicher Aufmerksamkeit. Die gegenwärtige Phase wird dabei durch einen erkennbaren paradigmatischen Wandel bestimmt, der sich am Leitbild einer integrierten ländlichen Entwicklung orientiert. Partizipation der lokalen Bevölkerung einerseits und Aufbau von Netzwerken relevanter öffentlicher, privater und ehrenamtlicher Akteure andererseits bilden den methodisch-instrumentellen Kern dieser Vorstellungen. Der Umbau wichtiger regionalpolitischer Institutionen Schottlands, die Implementierung mehrerer modellhafter Förderprogramme wie auch eine Reihe lokaler Initiativen "von unten“ illustrierten auf anschauliche Weise, welche Richtung bei der praktischen Umsetzung entsprechender Handlungsansätze eingeschlagen wurde.

Anhand der Ergebnisse eigener empirischer Untersuchungen (vgl. Anm. 1) ergibt sich ein ausgesprochen heterogenes Bild der neuen regionalpolitischen Landschaft Schottlands. Einige wesentliche Befunde können wie folgt zusammengefasst werden:

\subsection{Highlands and Islands Enterprise}

Mit der Einsetzung von HIE und ihrem Netz von zehn dezentralen Local Enterprise Companies (LEC) wurde ein institutioneller Rahmen geschaffen, der für die Regionalentwicklung von zentraler Bedeutung ist. Dies gilt zunächst für die territoriale Struktur von $H I E$, die die Highlands and Islands in Abgrenzung zu den übrigen Teilen des ländlichen Schottlands insgesamt als eigenes Programmgebiet definiert. Hierdurch wird ein hohes $\mathrm{Maß}$ an Aufmerksamkeit für die besonderen Probleme dieses Raumes sichergestellt. Zugleich erlaubt die dezentrale Struktur der LECs die Formulierung und Ausgestaltung von Förderpolitiken, die den spezifischen Erfordernissen der jeweiligen Regionen so weit als möglich angepasst werden können. Bestandteil dieser Politiken ist auch und gerade die Funktion der $L E C s$ als Mittler zwischen nationalen, regionalen und lokalen Agenden einerseits und zwischen öffentlichen, privaten und ehrenamtlichen Akteuren andererseits. Je nach Projektanforderung arbeiten HIE bzw. die LECs mit verschiedenen Partnern (Kommunen, Unternehmen, Dorfgemeinschaften, Bürgerinitiativen usw.) zusammen, wobei es sich zunächst häufig um informelle, projektgebundenene Partnerschaften handelt. Erklärtes Ziel ist es jedoch, diese in Zukunft stärker zu formalisieren, einerseits über die Installierung von sog. Local Economic Forums, lokalen Foren zur Abstimmung der Wirtschaftsförderung, anderer- seits über die zükünftige Implementierung des sog. Community Planning (vgl. Kap. 3.4).

So fällt HIE damit auch die Rolle eines Katalysators für die weitere Implementierung von Handlungsansätzen einer integrierten ländlichen Entwicklung $\mathrm{zu}$, dessen konzeptionelle Ausrichtung sich zumindest teilweise in der Programm- bzw. Organisationsstruktur von HIE widerspiegelt. Mit ihrer starken Präsenz in der Region sind HIE und die LECs in besonderer Weise berufen, die Vorstellungen integrierter Entwicklungsansätze in die Ausgestaltung der verschiedenen Agenden einzubringen bzw. dort zu unterstützen.

Die Defizite demokratischer Legitimierung und Kontrolle einer Quango stehen diesem Anspruch nur bedingt im Wege. Wie die jüngere Entwicklung zeigt, wurde insbesondere mit der Einrichtung der Local Economic Forums eine Möglichkeit geschaffen, die Aktivitäten von HIE und von kommunaler Wirtschaftsförderung sowie weiteren potenziellen Akteuren besser abzustimmen als bisher. Ein vollständiger Ausschluss von Konflikten kann damit gleichwohl nicht garantiert werden (vgl. Fairley/Lloyd 1998, S. 207 ff.).

\subsection{Modellvorhaben}

Im Untersuchungsgebiet kamen zwei zeitlich beschränkte, überregional initiierte Modellvorhaben zur Förderung der Regionalentwicklung in einigen besonders strukturschwachen Teilgebieten zur Anwendung: Duthchas (1998-2001) und Initiative at the Edge (zunächst 1998-2003, inzwischen verlängert). Beide Modellvorhaben an der äußersten Peripherie des Schottischen Hochlandes sind Ausdruck der Dynamik des ablaufenden Umgestaltungsprozesses, der weite Bereiche der Regionalpolitik erfasst hat und auch eine Reihe „Von oben" gesteuerter Handlungsansätze mit einschließt. Deren besonderer Wert liegt in der Initiierung mehrheitlich kleinräumig, teilweise lokal ausgerichteter Handlungsansätze, in deren Zentrum die Aktivierung und Partizipation der Bevölkerung in einigen der "most fragile areas“ ganz Schottlands steht. Einige dieser Gebiete sind mit den genannten Programmen erstmals in den Fokus regionalpolitischer Aktivitäten gerückt.

Dúthchas war ein Pilotprojekt, das ursprünglich auf eine Initiative der Naturschutz-Organisation Scottish National Heritage (SNH, wie HIE eine sog. Quango) zurückgeht. $S N H$ war Anfang der 1990er Jahre beauftragt worden, eine neue Schutzkategorie zu entwickeln, was jedoch aus verschiedenen Gründen nicht weiterverfolgt worden war. Das Highland Council Planning Department griff diese Idee wenig später auf und modifi- 
zierte unter Mitwirkung von zehn weiteren öffentlichen Einrichtungen, wie beispielsweise Western Isles Council, Highland Council, HIE, SNH, Western Isles Enterprise, Scottish Tourist Board, den Ansatz zu einer breiteren Strategie. Deren Zielsetzung war die Schaffung eines ,,affordable, transferable process and framework for addressing sustainable development and integrated land management in peripheral rural areas“ (Dúthchas 2002, S. 4). Das Neuartige an diesem Projekt war neben dem Nachhaltigkeitsansatz die räumliche, integrierte Herangehensweise an Entwicklung, wobei die speziellen Bedürfnisse eines definierten Teilraumes im Vordergrund stehen sollten. Die Frage nach den Bedürfnissen sollte sowohl von der lokalen Bevölkerung als auch von nationalen (schottischen) Institutionen und Interessensvertretern beantwortet werden. Im Vorfeld wählte das Highland Council Planning Department anhand statistischer Daten zunächst eine Reihe von Gebieten aus, welche die vorab festgelegten Kriterien erfüllten (u. a. die große Entfernung von Zentren, eine schwierige ökonomische Lage, hohe Abwanderungsraten sowie das Vorhandensein einer unverwechselbaren Landschaft und kulturellen Tradition). Aus diesen wurden schließlich unter Beteiligung von lokalen Mitarbeitern verschiedener Einrichtungen die drei „bedürftigsten“ Gebiete ausgewählt ${ }^{2}$.

Finanziert wurde Dúthchas durch das LIFE Programm der EU sowie durch die oben z.T. genannten elf Partner, inhaltlich beteiligt waren an dem Projekt jedoch insgesamt 19 öffentliche Einrichtungen sowie drei aus NGOs und privaten Interessensvertretern bestehende Netzwerke. Themen wie nachhaltige Land- und Küstennutzung, Produktion und Vermarktung lokaler Produkte, sanfter Tourismus, erneuerbare Energien, Transport und Infrastruktur sowie die Versorgung mit Dienstleistungen wurden von der lokalen Bevölkerung unter Anleitung sog. Area Coordinators und den beteiligten Partnern bearbeitet und Ziele in einem recht langwierigen Entscheidungsprozess abgestimmt. Genau hierin liegt auch das Dilemma von Dúthchas: Primär als grundsätzliches Modellprojekt für die Entwicklung nachhaltiger Strategien mit intensiver Partizipation aller Akteure gedacht, sind die Ergebnisse eher "theoretischer" Natur und liegen in Form von professionell erstellten Leitbildern und Aktionsplänen vor. Bei den lokalen Akteuren herrscht jedoch angesichts der Theorielastigkleit überwiegend Enttäuschung vor; sie hätten die entwickelten Projektideen lieber verwirklicht gesehen - was aber innerhalb der dreijährigen Laufzeit von Dúthchas nicht machbar war. Der praktische Wert von Dúthchas wird sich so erst in den nächsten Jahren herauskristallisieren, wenn ersichtlich ist, was die einzelnen Teilgebiete mit den von ihnen entwickelten Strategien konkret anfangen können.
Im gleichen Jahr wie Dúthchas wurde das Initiative at the Edge-Projekt durch Minister des damals noch existierenden Scottish Office initiiert, um den extrem peripheren und strukturschwachen Regionen der Highlands und Islands besondere Aufmerksamkeit und Unterstützung zukommen zu lassen. ${ }^{3}$ Die Initiative gründete sich auf der Erkenntnis, dass öffentlicher und privater Sektor in diesen Gebieten die gravierenden Probleme allein nicht bewältigen können und die lokale Bevölkerung in die Entwicklung von Lösungsstrategien einbezogen werden muss. Zentrale Prinzipien sind Partizipation, Einbeziehung aller wichtigen Institutionen und Nachhaltigkeit. Insofern ist Initiative at the Edge gedacht als ein Programm, das im Partnerschafts-Modell "Hilfe zur Selbsthilfe“ ermöglichen soll und in dessen Verlauf Ideen entwickelt, gebündelt und gewichtet werden sollen. Eine zentrale Rolle besitzt der Local Development Officer, der die Gruppen betreut und anleitet sowie Unterstützung bei Antragstellungen und Durchführung von Projekten leistet.

Nach der ersten Förderperiode 1998-2001 wurde das Programm unter Federführung des Scottish Executive Lifelong Learning Department (SELLD) fortgesetzt, wobei kein Gebiet länger als bis Ende 2003 gefördert werden sollte. Nach wie vor strittig ist die Gestaltung von möglichen Ausstiegsstrategien für die Gebiete, die Anfang 2004 aus der Förderung heraus gefallen sind. Unabhängig davon wurde jedoch eine Verlängerung des Programms beschlossen und werden zur Zeit (April 2004) die neuen Fördergebiete benannt. Finanziert wird Initiative at the Edge durch HIE, den Rural Challenge Fund, Scottish Executive (Social Inclusion Fund) und European Rural Development Fund (vgl. EKOS 2001), wobei in diesem Rahmen lediglich geringe Summen für den Anschub von Projekten zur Verfügung stehen. Für die Umsetzung der Projekte müssen weitere Förderquellen erschlossen werden.

Ganz im Gegensatz zu Dúthchas hatte Initiative at the $E d g e$ beim Projektstart keine übergeordnete, langfristige Strategie, was sich damit erklären lässt, dass die Initiative auf das "private" Engagement weniger Politiker zurückzuführen ist. So gab es keine an objektive Daten angelegten Auswahlkriterien für die Zielgebiete und keine Schulungen für die Local Development Officers. Dennoch ist es grösstenteils gelungen, lokale Gruppen zu bilden und Projekte in den unterschiedlichsten Handlungsfeldern zu definieren bzw. zu initiieren (für Beispiele siehe Tab.1).

Die Erfahrungen insbesondere mit der Initiative at the Edge haben gezeigt, dass es nicht unproblematisch ist, die lokale Ebene ohne Einbindung in ein übergeordnetes Konzept und ohne konkrete Zielvorstellungen zur Selbstinitiative anzuregen. Wenn es ein erklärtes Ziel 
Ingo Mose und Yvonne Brodda: Neue regionale Entwicklungskonzepte für periphere ländliche Räume

Ausgewählte Projekte der Initiative at the Edge im Programmgebiet Bays of Harris

\begin{tabular}{|ll|}
\hline Arbeitsmarkt & - Einrichtung einer Service-Station für die Anlandung und Verpackung von Krabben \\
& - Adaptierung einer alten Schule als Bürogebäude \\
& - Unterstützung lokaler Gruppen bei der Einwerbung von Fördergeldern \\
\hline Tourismus & - Ersterung von Nutzungsmöglichkeiten für den Einsatz erneuerbarer Energien \\
& - Restaurierung einer alten Mühle und Umnutzung als Informationszentrum \\
& - Restaurierung von alten croft-Häusern für die Nutzung als Ferienhäuser
\end{tabular}

Quelle: Initiative at the Edge (2001): Bays of Harris Action Programme. April 2002 - March 2005. - o.O.

der Regionalpolitik ist, periphere Regionen nachhaltig zu stärken, müssen seitens der zuständigen Ministerien für das jeweilige Programm klare Vorgaben formuliert werden. Diese erlauben es einerseits, den lokalen Akteuren klar zu machen, welche Erwartungen an sie gestellt werden, und andererseits die involvierten Institutionen und Behörden zur Unterstützung der lokalen Initiativen und Netzwerke zu verpflichten. Sonst wird der eigene Geschäftsplan den Institutionen wie HIE wichtiger bleiben und kein Bewusstsein über die Probleme der lokalen Ebene entstehen können. Dúthchas war in dieser Hinsicht von Beginn an durchdachter, hat jedoch durch die Theorielastigkeit und umständlichen Entscheidungsprozesse die Mitwirkenden überfordert. Das zeigt eindrucksvoll, dass praktische Ergebnisse und Erfolgserlebnisse für die Motivation der Betroffenen unbedingt erforderlich sind. Letztendlich kommt es darauf an, die richtige Balance - vor allem auch in zeitlicher Hinsicht - zu finden: zwischen Strategieentwicklung und Planung auf der einen Seite sowie Handeln und Projektumsetzung auf der anderen Seite.

\subsection{Lokale Initiativen}

Zusätzlich zu den „von oben“ initiierten Modellversuchen ist heute in allen Teilen der Highlands and Islands eine breite Aktivierung „von unten“ anzutreffen. Diese ist Ausdruck einer wachsenden Bereitschaft unter der lokalen Bevölkerung, sich aktiv und engagiert in unterschiedliche Prozesse der örtlichen und überörtlichen Entwicklung einzubringen und Einfluss auf die praktische Ausgestaltung entsprechender Agenden zu nehmen. Dies gilt sowohl für die Möglichkeiten der Partizipation im Rahmen von vorstrukturierten Beteiligungsverfahren bestimmter Förderprogramme als auch auf der Basis von Eigeninitiative und Selbsthilfe außerhalb von Programmen.

Zielsetzungen und Arbeitsweise der untersuchten Initiativen im Rahmen der Fallstudie korrespondieren in hohem Maße mit den konzeptionellen Vorstellungen einer integrierten ländlichen Entwicklung: Sie basieren auf der Aktivierung loser oder fester Formen der Zusammenarbeit unterschiedlicher lokaler Akteure, die gemeinsam Träger spezifischer, sektorübergreifend angelegter Projektvorhaben in diversen Handlungsfeldern werden.

Als ein grundsätzliches Problem jeglicher Formen von lokalen Initiativen erweist sich dabei die Sicherstellung adäquater und ausreichender Ressourcen. Weniger betrifft dies die Gewährung investiver Mittel als vielmehr die Ausstattung mit personellen Ressourcen für Beratung, Begleitung und Betreuung der Projektarbeit. Erfolgreiche Entwicklung und Umsetzung lokaler Agenden „von unten“, die Sicherung einer hohen Qualität 
sowie die Kontinuität von eingeleiteten Entwicklungsprozessen sind unverzichtbar an entsprechende Hilfen in Form professioneller Development Officers, Community Officers o.ä. gebunden.

\subsection{Fazit}

Abschließend bleibt festzuhalten, dass der "neuen" regionalpolitischen Landschaft Schottlands eine abgestimmte Strategie (noch) fehlt. Ein streamlining von Programmen, nationaler Prioritätensetzung sowie systematischer Beteiligung von lokalen und regionalen Akteuren ist bislang nur in Ansätzen vorhanden. So ist die fehlende Bereitschaft von Institutionen und Behörden, sich wirklich auf Kooperationsprozesse mit nicht institutionalisierten Akteursgruppen einzulassen, mit fehlenden Rahmenbedingungen für die Koordinierung und Abstimmung der Partnerschaften auf der nationalen Ebene zu erklären. „Working in partnerships" ist zwar offizielles Postulat und in den meisten Projektzusammenhängen „vor Ort“ längst Realität, aber dennoch "nur" informeller Natur. Spannungen und Differenzen treten vor allem bei unterschiedlicher Prioritätensetzung auf nationaler und lokaler Ebene auf sowie zwischen strategischem Planungsanspruch einerseits und lokalem Engagement andererseits.

Dasselbe wie für die vertikale lässt sich auch für die horizontale, sektorübergreifende Integration konstatieren: Auch hier wird integrierte ländliche Entwicklung postuliert, findet ad hoc in Projekten auf regionaler und lokaler Ebene bereits statt, während auf nationaler Ebene nach wie vor eine große Lücke zwischen Theorie und Praxis besteht. So macht der Anteil für die Local Rural Partnerships beispielsweise nur etwa 2-3\% der Gesamtausgaben für ländliche Entwicklung aus - der Löwenanteil geht nach wie vor in die Landwirtschaftsförderung. Kritischer Punkt ist, effiziente Mechanismen für die Integration von noch auszuwählenden Politikfeldern zu entwickeln, die für integrierte ländliche Entwicklung relevant sind. Dabei müssten vor allem zwei Hemmnisse überwunden werden: der große politische Einfluss der Agrarlobby innerhalb der schottischen Verwaltung sowie die europäische Agrarpolitik, die sich dem schottischen Einfluss freilich entzieht.

Ein Versuch, die verschiedenartigen Ansätze miteinander in Beziehung zu setzen und zu integrieren, ist das oben bereits genannte Community Planning, das im Rahmen des Local Government in Scotland Act 2003 gesetzlich verankert wurde und zum 1. April 2003 in Kraft getreten ist. Zum gegenwärtigen Zeitpunkt können noch keine Aussagen zur Umsetzung oder zu den Auswirkungen auf die Regionalpolitik insgesamt ge- troffen werden. Gemäß den Absichtserklärungen soll das Instrument des Community Planning Koordinierungsbasis für alle Partnerships sein und für ein strategisches streamlining sorgen. Während die lokalen und regionalen Initiativen wie auch der Modellversuch Initiative at the Edge Ansätze mit eher räumlichem Bezug sind, soll das Community Planning den strategischen Rahmen setzen. Dabei soll es sowohl der horizontalen - zwischen den verschiedenen Politikfeldern - als auch der vertikalen - zwischen Bevölkerung, Institutionen und Regierung - Integration dienen. Es bleibt abzuwarten, inwieweit diese Erwartungen eingelöst werden können und damit auch eine nachhaltige Stärkung lokaler Agenden und ihres Beitrags zur Entwicklung der Highlands and Islands verbunden sein wird.

\section{Anmerkung}

(1)

Der Beitrag beruht auf ersten Ergebnissen eines DFG-Projektes (2001-2004) zur vergleichenden Untersuchung ausgewählter Fallstudien in Europa. Neben dem Gebiet des Western Isles Councils sowie des ehemaligen Districts Skye and Lochalsh in Schottland sind dies Jämtlands Län in Schweden sowie die Region Eisenwurzen in Österreich. Näheres siehe in RuR 3-4.2004.

(2)

Als Teilgebiete ausgewählt wurden North Uist, eine Insel der Western Isles, die Halbinsel Trotternish auf der Insel Skye sowie - außerhalb des Untersuchungsgebietes - North Sutherland im Nordwestern des Hochlandes.

(3)

Als Teilgebiete ausgewählt wurden Uig \& Bernera auf der Insel Lewis, Bays of Harris auf der Insel Harris, Lochboisdale \& Eriskay auf den Uists sowie - außerhalb des Untersuchungsgebietes Ardnamurchan, Colonsay, North Sutherland und Westray/Papa Westray auf den Orkney Inseln.

\section{Literatur}

Black, S.; Conway, E. (1996): The European Community's LEADER Programme in the Highlands and Islands. In: Scottish Geographical Magazine 112, No. 2, S. 101-106

Brown, A. (2000): Evaluation of the Local Rural Partnership Scheme. Executive Summary. - Edinburgh

Dúthchas (2002): Our Place in the Future. Final Report. - Inverness

EKOS Limited (2001): Interim Evaluation of Initiative at the Edge I Iomairt aig an oir. Final Report. - Inverness

Fairly, J.; Lloyd, G. (1998): Enterprise in Scotland. AMID-Term Assessment of an Institutional Innovation for Economic Development. In: Halkier, H.; Danson, M.; Damborg, C. (Eds.): Regio- 
nal Development Agencies in Europe. = Regional Policy and Development Series 21. London, Philadelphia, S. 199-212

Greenwood, J.; Pyper, R.; Wilson, D. (2002): New Public Administration in Britain. $3^{\text {rd }}$. edition. - London.

Heineberg, H. (1997): Großbritannien. Raumstrukturen, Entwicklungsprozesse, Raumplanung. 2 . Auflage. $=$ Perthes Länderprofile. - Gotha

Hunter, J. (2000). Last of the Free. A Millennial History of the Highlands and Islands of Scotland. - Edinburgh

Initiative at the Edge (2001): Bays of Harris Action Programme. April 2002 - March 2005. - o.O.

Marsden, T.; Bristow, G. (2000): Progressing Integrated Rural Development: A Framework for Assessing the Integrative Potential of Sectoral Policies. In: Regional Studies 34, No. 5, S. 455-469

Shortall, S.; Shucksmith, M. (1998): Integrated rural development: Issues arising from the Scottish experience. In: European Planning Studies 6, No. 1, S. 73-88

Shucksmith, M. (1998): Rural and Regional Policy Implementation: Issues Arising from the Scottish Experience. Manuscript at the Arkleton Research Center. - Aberdeen
Turnock, D. (1974): Scotland's Highlands and Islands. = Problem Regions of Europe. - Oxford

Wehling, H.-W. (2000): Tourismus in Schottland. Historische Entwicklungen und Images, aktuelle Strukturen und Marketingstrategien. In: Geographische Rundschau 52, H. 1, S. 27-34

Ausgewählte Internetadressen:

www.hie.co.uk

www.initiative-at-the-edge.org.uk

www.duthchas.org.uk

www.crofterscommission.org.uk

www.snh.org.uk

Prof. Dr. Ingo Mose

Hochschule Vechta

Institut für Umweltwissenschaften

Oldenburger Straße 97

49377 Vechta

E-Mail: ingo.mose@uni-vechta.de

Dipl.-Geogr. Yvonne Brodda

Hochschule Vechta

Institut für Umweltwissenschaften

Oldenvburger Straße 97

49377 Vechta

E-Mail: ybrodda@iuw.uni-vechta.de 